\title{
BOUNDS OF GENERALIZED RELATIVE OPERATOR ENTROPIES
}

\author{
ISMAIL NiKOUfAR AND MEHDi ALINEJAD
}

Abstract. In this paper, we identify upper and lower bounds of the generalized relative operator entropy based on the notion of perspectives. Moreover, we find upper and lower bounds of the Tsallis relative operator entropy to specify the bounds of the relative operator entropy.

Mathematics subject classification (2010): 47A63, 46L05, 46L60.

Keywords and phrases: Operator inequality, operator Shannon type inequality, relative operator entropy, generalized relative operator entropy.

\section{REFERENCES}

[1] S. ABE, Monotonic decrease of the quantum nonadditive divergence by projective measurements, Phys. Lett. A, 312 (2003), 336-338, and its Corrigendum, 324, (2004), pp. 507.

[2] S. S. DRAGOMIR, Further inequalities for relative operator entropy, RGMIA Res. Rep. Col 1. 18, Art. 160 (2015), [http://rgmia.org/papers/v18/v18a160.pdf].

[3] S. S. DRAGOMIR, Some inequalities for relative operator entropy, Preprint RGMIA Res. Rep. Col 1. 18, Art. 145 (2015) [http://rgmia.org/papers/v18/v18a145.pdf].

[4] S. S. DRAGOMIR, Bounds for the normalized Jensen functional, Bull. Austral. Math. Soc., 74 (2006), 471-478.

[5] A. Ebadian, I. Nikoufar, And M. Eshagi Gordi, Perspectives of matrix convex functions, Proc. Natl. Acad. Sci., 108, 18 (2011), 7313-7314.

[6] E. G. EfFros, A matrix convexity approach to some celebrated quantum inequalities, Proc. Natl. Acad. Sci. U S A., 106, 4 (2009), 1006-1008.

[7] T. FURUTA, Parametric extensions of Shannon inequality and its reverse one in Hilbert space operators, Linear Algebra Appl., 381 (2004), 219-235.

[8] T. FURUTA, Reverse inequalities involving two relative operator entropies and two relative entropies, Linear Algebra Appl., 403 (2005), 24-30.

[9] T. FURUTA, Two reverse inequalities associated with Tsallis relative operator entropy via generalized Kantorovich constant and their applications, Linear Algebra Appl., 412, 2-3 (2006), 526-537.

[10] J. I. FuJII AND E. KAMEI, Relative operator entropy in noncommutative information theory, Math. Japonica, 34 (1989), 341-348.

[11] J. I. FUJII AND E. KAMEI, Uhlmann's interpolational method for operator means, Math. Japonica, 34 (1989), 541-547.

[12] S. FurUichi, K. YANAGI, K. KURIYAma, A note on operator inequalities of Tsallis relative operator entropy, Linear Algebra Appl., 407 (2005), 19-31.

[13] F. Kubo And T. Ando, Means of positive linear operators, Math. Ann., 246 (1979-1980), 205-224.

[14] G. LindBlad, Completely positive maps and entropy inequalities, Commun. Math. Phys., 40 (1975), $147-151$.

[15] M. OHYA AND D. PetZ, Quantum entropy and its use, Springer-Verlag, Heidelberg, 1993, Second edition, 2004.

[16] I. Nikoufar, A. Ebadian, AND M. Eshagi Gordi, The simplest proof of Lieb concavity theorem, Adv. Math., 248 (2013), 531-533.

[17] I. NikoufaR, On operator inequalities of some relative operator entropies, Adv. Math., 259 (2014), $376-383$.

[18] I. NiKoufar, Operator versions of Shannon type inequality, Math. Ineq. Appl., 19, 1 (2016), 359367. 
[19] I. NiKoufaR, A perspective approach for characterization of Lieb concavity theorem, Demonstratio Math., 49, 4 (2016), 463-469.

[20] C. Ts allis, Possible generalization of Bolzmann-Gibbs statistics, J. Stat. Phys., 52 (1988), 479-487.

[21] K. Yanagi, K. Kuriyama, And S. FurUichi, Generalized Shannon inequalities based on Tsallis relative operator entropy, Linear Alg. Appl., 394 (2005), 109-118.

[22] H. UMegaKi, Conditional expectation in an operator algebra, IV (entropy and information), Kodai Math. Sem. Rep., 14 (1962), 59-85.

[23] L. Zou, Operator inequalities associated with Tsallis relative operator entropy, Math. Ineq. Appl., 18, 2 (2015), 401-406. 